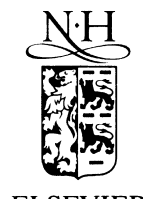

Nuclear Instruments and Methods in Physics Research B 173 (2001) 332-343

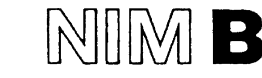

Beam Interactions

with Materials \& Atoms

\title{
Improved electron transport mechanics in the PENELOPE Monte-Carlo model
}

\author{
A.F. Bielajew ${ }^{a, *}$, F. Salvat ${ }^{b}$ \\ a Department of Nuclear Engineering and Radiological Sciences, University of Michigan, 1906 Cooley Building, 2355 Bonisteel Boulevard, \\ Ann Arbor, MI 48109-2104, Michigan, USA \\ ${ }^{\mathrm{b}}$ Facultat de Física (ECM), Universitat de Barcelona, Societat Catalana de Física, Diagonal 647, 08028 Barcelona, Spain
}

Received 17 March 2000

\begin{abstract}
We describe a new model of electron transport mechanics, the method by which an electron is transported geometrically in an infinite medium as a function of pathlength, $s$, the accumulated elastic multiple-scattering angular deflection characterized by $\Theta(s)$, the polar scattering angle, and $\Phi$, a random azimuthal angle. This model requires only one sample of the multiple-scattering angle yet it reproduces exactly the following spatial moments and space-angular correlations: $\langle z\rangle,\langle x \sin \Theta \cos \Phi\rangle,\langle y \sin \Theta \sin \Phi\rangle,\langle z \cos \Theta\rangle,\left\langle x^{2}\right\rangle,\left\langle y^{2}\right\rangle$ and $\left\langle z^{2}\right\rangle$. Moreover, the distributions associated with these moments exhibit a good improvement over the PENELOPE transport mechanics model when compared selfconsistently with the results of analog simulations. When we split the transport step into two steps with equal pathlength, we observe excellent agreement with the distributions, indicating that the algorithm nearly matches higher order moments when employed in this way. The equations described herein are relatively inexpensive to employ in an iterative Monte-Carlo code. We have employed the new model to demonstrate the usefulness of the new mechanics for several examples that span the dynamic range of application. (c) 2001 Elsevier Science B.V. All rights reserved.
\end{abstract}

PACS: $02.50 \mathrm{Ng} ; 13.60 \mathrm{Fz} ; 25.30 \mathrm{Bf} ; 34.80 \mathrm{Bm}$

Keywords: Monte-Carlo simulation; Condensed history; Elastic scattering; Multiple-scattering; Coulomb scattering

\section{Introduction}

One of the most challenging problems in the Monte-Carlo simulation of high-energy electron (and positron) transport is the generation of spa-

\footnotetext{
${ }^{*}$ Corresponding author. Tel.: +1-734-7646364; fax: +1-7347634540 .

E-mail address: bielajew@umich.edu (A.F. Bielajew).
}

tial displacements of the particle. In each step of the simulation, the electron is moved a certain pathlength, $s$, through the medium. The angular deflection after this pathlength is determined by the polar multiple-scattering angle, $\Theta(s)$, and the azimuthal angle $\Phi$, which is distributed uniformly on $[0,2 \pi]$. For a given elastic cross-section, the theory of Goudsmit and Saunderson [1,2] provides the multiple-elastic scattering distribution from which $\Theta(s)$ can be sampled. The difficulty comes 
from the fact that the space displacement $(x, y, z)$ at the end of the step, although strongly correlated with the angular deflection, is not known. Formal solutions of the transport equation $[3,4]$ provide closed expressions only for the moments of the space displacements and space-angle correlations. Since only a few of these moments can be evaluated and employed in a practical transport scheme, there is not enough information to characterize $(x, y, z)$ unambiguously. The prescription that relates $(x, y, z)$ to $\Theta(s), \Phi$ and $s$ will be called the "electron transport mechanics" [4].

The PENELOPE Monte-Carlo code system [57], a general purpose coupled $\mathrm{e}^{ \pm} \gamma$ Monte-Carlo code, employs a "random hinge" electron transport mechanics' scheme that can be summarized as follows:

$x / s=r \sin \Theta(s) \cos \Phi$,

$y / s=r \sin \Theta(s) \sin \Phi$,

$z / s=(1-r)+r \cos \Theta(s)$,

where $r$ is a random number sampled uniformly on $[0,1]$, and the pathlength, $s$, for which the multiplescattering angle $\Theta(s)$ is calculated and interpreted as the total curved pathlength that the electron travels through the medium.

Although this scheme is an ansatz, it produces high quality results as indicated through compliance [4] with Lewis' moments [3]. The Lewis moments studied in the previous work were

$$
\begin{aligned}
& \langle z\rangle=\int_{0}^{s} \mathrm{~d}^{\prime} \mathrm{e}^{-g_{1} s^{\prime}} \\
& \langle z \mu\rangle=\frac{1}{3} \int_{0}^{s} \mathrm{~d} s^{\prime} \mathrm{e}^{-g_{1}\left(s-s^{\prime}\right)}\left(1+2 \mathrm{e}^{-g_{2} s^{\prime}}\right) \\
& \langle x \sin \Theta \cos \Phi+y \sin \Theta \sin \Phi\rangle \\
& \quad=\frac{2}{3} \int_{0}^{s} \mathrm{~d} s^{\prime} \mathrm{e}^{-g_{1}\left(s-s^{\prime}\right)}\left(1-\mathrm{e}^{-g_{2} s^{\prime}}\right) \\
& \left\langle z^{2}\right\rangle=\frac{1}{3} \int_{0}^{s} \mathrm{~d} s^{\prime} \int_{0}^{s^{\prime}} \mathrm{d} s^{\prime \prime} \mathrm{e}^{-g_{1}\left(s^{\prime}-s^{\prime \prime}\right)}\left(1+2 \mathrm{e}^{-g_{2} s^{\prime \prime}}\right) \\
& \left\langle x^{2}+y^{2}\right\rangle=\frac{2}{3} \int_{0}^{s} \mathrm{~d} s^{\prime} \int_{0}^{s^{\prime}} \mathrm{d} s^{\prime \prime} \mathrm{e}^{-g_{1}\left(s^{\prime}-s^{\prime \prime}\right)}\left(1-2 \mathrm{e}^{-g_{2} s^{\prime \prime}}\right)
\end{aligned}
$$

where the $g$ s are moments of the single-scattering cross-section $\sigma(\mu)$ with Legendre polynomials,

$$
g_{\ell}=2 \pi \frac{N_{\mathrm{A}} \rho}{A} \int_{0}^{s} \mathrm{~d} s^{\prime} \int_{-1}^{1} \mathrm{~d} \mu \sigma(\mu)\left[1-P_{\ell}(\mu)\right],
$$

in which $N_{\mathrm{A}}$ is Avogadro's number, $A$ is atomic weight (we assume single-element medium) and $\rho$ is the density of the medium. Here, the distance $s$ is expressed as a unit of length and the integration variable $\mu$ is the cosine of the polar scattering angle. Since the scattering model we are considering is azimuthally symmetric, $\langle x \sin \Theta \cos \Phi\rangle=\langle y \sin \Theta$ $\sin \Phi\rangle,\left\langle x^{2}\right\rangle=\left\langle y^{2}\right\rangle$, and are combined in Eq. (2).

The angular distribution after a pathlength, $s$, is given by

$f(\mu ; s)=\sum_{l=0}^{\infty}\left(l+\frac{1}{2}\right) \mathrm{e}^{-s g_{\ell}} P_{\ell}(\mu)$

and we also have

$$
\langle\mu\rangle=\mathrm{e}^{-g_{1} s}, \quad\left\langle\mu^{2}\right\rangle=\frac{1+2 \mathrm{e}^{-g_{2} s}}{3} .
$$

Here, we have ignored the energy dependence of the single-scattering cross-section, which allows for greater analytic development. The above equations may be expressed in energy-dependent form, for example, employing the continuous slowing down approximation (CSDA), whereby the integrals over pathlength, $s$, in Eq. (2) are replaced by integrals over energy, and pathlength and energy are related through a stopping-power relationship. We leave this, or similar, adaptations to future work. Having ignored energy loss, the integrals in Eq. (2) may be performed with the result

$$
\begin{aligned}
& \frac{\langle z\rangle}{s}=\frac{1-\mathrm{e}^{-\xi}}{\xi}, \\
& \frac{\langle z \mu\rangle}{s}=\frac{1}{3 \xi}\left(1-\mathrm{e}^{-\xi}+2 \frac{\mathrm{e}^{-\xi}-\mathrm{e}^{-\gamma \xi}}{\gamma-1}\right), \\
& \frac{\langle x \sin \Theta \cos \Phi+y \sin \Theta \sin \Phi\rangle}{s}=\frac{2}{3 \xi}\left(1+\frac{\mathrm{e}^{-\gamma \xi}-\gamma \mathrm{e}^{-\xi}}{\gamma-1}\right), \\
& \frac{\left\langle z^{2}\right\rangle}{s^{2}}=\frac{2}{3 \gamma \xi^{2}}\left(\gamma\left(\xi+\mathrm{e}^{-\xi}\right)-(\gamma-2)+2 \frac{\mathrm{e}^{-\gamma \xi}-\gamma \mathrm{e}^{-\xi}}{\gamma-1}\right), \\
& \frac{\left\langle x^{2}+y^{2}\right\rangle}{s^{2}}=\frac{4}{3 \gamma \xi^{2}}\left(\gamma \xi-(\gamma+1)-\frac{\mathrm{e}^{-\gamma \xi}-\gamma^{2} \mathrm{e}^{-\xi}}{\gamma-1}\right),
\end{aligned}
$$

where $\xi=s g_{1}$ and $\gamma=g_{2} / g_{1}$ which spans the range from 0 (backward scattering) to 3 (high energy, 
forward directed) and has the value unity for isotropic scattering.

Generally, much of the range of application involves small values of $\xi$ which suggests an expansion of the moments in a Taylor series in $\xi$. These results have been stated elsewhere [4], but we include them here for completeness and for the next order in $\xi$ for later use. To $\mathrm{O}\left(\xi^{3}\right)$,

$$
\begin{aligned}
& \frac{s-\langle z\rangle}{s}=\frac{\xi}{2}-\frac{\xi^{2}}{6}+\frac{\xi^{2}}{24}, \\
& \frac{\operatorname{cov}(z, \mu)}{s}=\frac{3-\gamma}{3} \xi+\frac{\gamma^{2}+\gamma-9}{9} \xi^{2}-\frac{\gamma^{3}+\gamma^{2}+\gamma-21}{36} \xi^{3}, \\
& \frac{\langle x \sin \Theta \cos \Phi+y \sin \Theta \sin \Phi\rangle}{s} \\
& =\frac{\gamma}{3} \xi-\frac{\gamma(\gamma+1)}{9} \xi^{2}+\gamma \frac{\gamma^{2}+\gamma+1}{36} \xi^{3}, \\
& \frac{\operatorname{var}(z)}{s^{2}}=\frac{2(3-\gamma)}{9} \xi+\frac{\gamma^{2}+\gamma-9}{18} \xi^{2}-\frac{\gamma^{3}+\gamma^{2}+\gamma-21}{90} \xi^{3}, \\
& \frac{\left\langle x^{2}+y^{2}\right\rangle}{s^{2}}=\frac{2 \gamma}{9} \xi-\frac{\gamma(\gamma+1)}{18} \xi^{2}+\gamma \frac{\gamma^{2}+\gamma+1}{90} \xi^{3},
\end{aligned}
$$

where $\operatorname{cov}(z, \mu)=\langle z \mu\rangle-\langle z\rangle\langle\mu\rangle$ and $\operatorname{var}(z)=\left\langle z^{2}\right\rangle-$ $\langle z\rangle^{2}$. We note that the $\mathrm{O}(\xi)$ terms of $\operatorname{cov}(z, \mu)$ and $\operatorname{var}(z)$ vanish in the high-energy limit. This will have interesting consequences as we shall see later on.

With the above information, we can compare predictions of spatial and angular moments of PENELOPEs transport mechanics. As shown previously [4], to $\mathrm{O}\left(\xi^{2}\right)$,

$$
\begin{aligned}
& \frac{\langle z\rangle_{\mathrm{P}}-\langle z\rangle}{\langle z\rangle}=\frac{\xi^{2}}{12}, \\
& \frac{\langle z \mu\rangle_{\mathrm{P}}-\langle z \mu\rangle}{\langle z \mu\rangle}=\frac{2 \gamma^{2}-4 \gamma+3}{36} \xi^{2}, \\
& \frac{\langle x \sin \Theta \cos \Phi+y \sin \Theta \sin \Phi\rangle_{\mathrm{P}}}{\langle x \sin \Theta \cos \Phi+y \sin \Theta \sin \Phi\rangle}-1 \\
& =-\frac{\gamma-2}{6} \xi+\frac{\gamma^{2}-\gamma+1}{36} \xi^{2}, \\
& \frac{\left\langle z^{2}\right\rangle_{\mathrm{P}}-\left\langle z^{2}\right\rangle}{\left\langle z^{2}\right\rangle}=\frac{3+2 \gamma(\gamma-1)}{36} \xi^{2}, \\
& \frac{\left\langle x^{2}+y^{2}\right\rangle_{\mathrm{P}}-\left\langle x^{2}+y^{2}\right\rangle}{\left\langle x^{2}+y^{2}\right\rangle}=-\frac{\gamma-1}{4} \xi+\frac{3-12 \gamma+13 \gamma^{2}}{240} \xi^{2} .
\end{aligned}
$$

We note, in particular, that the lateral moments, $\langle x \sin \Theta \cos \Phi+y \sin \Theta \sin \Phi\rangle_{\mathrm{P}}$ and $\left\langle x^{2}+y^{2}\right\rangle_{\mathrm{P}}$ have $\mathrm{O}(\xi)$ discrepancies, while the longitudinal ones shown are $\mathrm{O}\left(\xi^{2}\right)$. This forms the main motivation for attempting to improve the model. We will see that our new form is able to reproduce the exact moments shown above.

\section{The improved model}

It suffices to say that we attempted many schemes before settling on the following model:

$$
\begin{aligned}
& x / s=f r \sin \Theta(s) \cos \Phi_{1}+\sigma \cos \Theta(s) \cos \Phi_{2}, \\
& y / s=f r \sin \Theta(s) \sin \Phi_{1}+\sigma \cos \Theta(s) \sin \Phi_{2}, \\
& z / s=k(1-r)+c+(k r+d) \cos \Theta(s),
\end{aligned}
$$

where $r$ is a random number sampled uniformly on $[0,1]$, and $f, \sigma, k, c$ and $d$ are constants independent of $r, \Theta$ and the $\Phi_{i}$ s. $\Phi_{1}$ and $\Phi_{2}$ are sampled uniformly on $[0,2 \pi]$ and are independent of each other. On average, $\Phi_{1}$ is associated with the random azimuthal direction of scattering after pathlength, $s$, while $\Phi_{2}$ provides some additional straggling about this direction.

In the analysis associated with the present work, it became apparent that the simple PENELOPE picture of a particle traveling a certain random distance, scattering and traveling the remainder of the pathlength, would have to be abandoned. Yet, the anticorrelation of the two longitudinal parts of $k$ (with and without $\cos \Theta(s)$ ) would have to be nearly preserved except for the modification by $c$ and $d$. The correlation between the $\cos \Theta(s)$-dependent part of $z$ and the lateral deflection is also important but the correlation is broken to some degree by the different modifying factors, $k$ and $f$. Physically, the $\sigma$-factor was motivated to break the absolute correlation between the azimuthal direction of scatter and the azimuthal direction of transport. This effect was recognized in the algorithm described by Kawrakow [8] although our approach is different. The $\cos \Theta(s)$-factor modifying the $\sigma$-term was found to be necessary to reduce some overprediction of the tail in lateral straggling distributions. 
The requirement that the model Eq. (9) reproduce the five exact Lewis moments given in Eq. (6) allows us to solve for $f, \sigma, k, c$ and $d$ with the result

$f=2 \frac{\langle x \sin \Theta \cos \Phi+y \sin \Theta \sin \Phi\rangle}{1-\left\langle\mu^{2}\right\rangle}$,

$\sigma=\sqrt{\frac{\left\langle x^{2}+y^{2}\right\rangle-\frac{f^{2}}{3}\left(1-\left\langle\mu^{2}\right\rangle\right)}{\left\langle\mu^{2}\right\rangle}}$,

$k=\sqrt{12 \frac{\operatorname{var}(z) \operatorname{var}(\mu)-\operatorname{cov}^{2}(z, \mu)}{\operatorname{var}^{2}(\mu)+\operatorname{var}(\mu)(1-\langle\mu\rangle)^{2}}}$,

$c=\langle z\rangle-\langle\mu\rangle \frac{\operatorname{cov}(z, \mu)}{\operatorname{var}(\mu)}-\frac{k}{2}$,

$d=\frac{\operatorname{cov}(z, \mu)}{\operatorname{var}(\mu)}-\frac{k}{2}$

The small $\xi$ behavior of Eqs. (10)-(14) is

$$
\begin{aligned}
f= & 1+\frac{\gamma-2}{6} \xi-\frac{\gamma-1}{12} \xi^{2}, \\
\sigma= & \frac{(5-\gamma) \gamma}{54} \xi^{2}, \\
k= & 1-\frac{2 \gamma-3}{4(3-\gamma)} \xi \\
& -\frac{12 \gamma^{4}-104 \gamma^{3}+176 \gamma^{2}-228 \gamma+171}{480(3-\gamma)^{2}} \xi^{2}, \\
c= & \frac{2 \gamma^{2}+2 \gamma-9}{24(3-\gamma)} \xi \\
& +\frac{12 \gamma^{4}-104 \gamma^{3}+16 \gamma^{2}+252 \gamma-189}{960(3-\gamma)^{2}} \xi^{2}, \\
d= & -\frac{2 \gamma^{2}-10 \gamma+9}{24(3-\gamma)} \xi \\
& +\frac{4 \gamma^{4}-8 \gamma^{3}-48 \gamma^{2}+84 \gamma-63}{320(3-\gamma)^{2}} \xi^{2},
\end{aligned}
$$

which is expressed above to $\mathrm{O}\left(\xi^{2}\right)$. The apparent singularity of these expressions is an artifact of leading order terms in $\xi$ vanishing in the limit $\gamma \rightarrow 3$. All this means that the series expansion expressed in Eq. (15) is of limited use. For numerical calculations we will have to resort to Eqs. (10)-(14) and Eq. (6) for most cases except very close to $\xi=0$ where rational expressions of two Taylor series in $\xi$ were employed.
The $\gamma \rightarrow 3$ limit of Eqs. (10)-(14) is

$f=1+\frac{1}{6} \xi-\frac{1}{6} \xi^{2}$

$\sigma=\frac{1}{9} \xi^{2}$

$k=\frac{1}{\sqrt{3}}\left(1+\frac{\xi}{5}+\frac{19}{1800} \xi^{2}\right)$,

$c=\frac{4-\sqrt{3}}{6}-\frac{(20+3 \sqrt{3})}{90} \xi+\left(\frac{2}{27}-\frac{19}{3600 \sqrt{3}}\right) \xi^{2}$,

$d=\frac{2-\sqrt{3}}{6}-\frac{(3 \sqrt{3}-5)}{90} \xi-\left(\frac{1}{54}+\frac{19}{3600 \sqrt{3}}\right) \xi^{2}$.

The "exact" forms of the five factors, $f, \sigma, k, c$ and $d$ are plotted in Figs. $1-5$ over the ranges $0 \leqslant \xi \leqslant 1$ and $0 \leqslant \gamma \leqslant 3$ - sufficient for any practical simulation. These should be contrasted to the standard PENELOPE model, $f=k=1, \sigma=c=d=0$. All the surfaces are flat with the most structure being exhibited near $\gamma=3$ which may have been expected from the previous discussion.

\section{Implementation of the new algorithm}

To generate random electron trajectories, the transport mechanics algorithm needs to be supplied with polar deflections, $\Theta(s)$, sampled from appropriate multiple-scattering distributions. Ultimately, the reliability of the simulation is governed by the physical quality of the singlescattering model adopted and the numerical accuracy of the pre-calculated multiple-scattering angular distribution. In the limit of small pathlengths, Larsen has shown [9] that condensed simulation should reproduce the exact solution of the transport equation, independently of the form of the underlying elastic cross-section. However, it has been argued [10] that the multiple-scattering algorithm must also be "robust". That is, the simulated spatial and angular distributions after a given pathlength should be the same no matter how that total pathlength is subdivided into substeps, each with its own deflection and displacement. A robust multiple-scattering algorithm has 


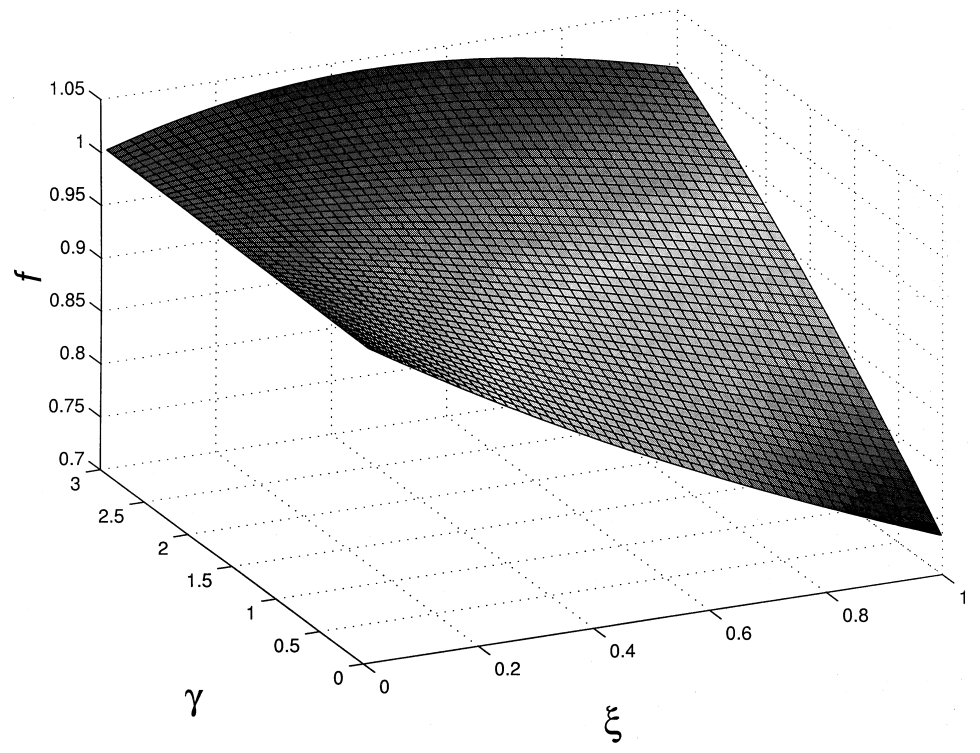

Fig. 1. $f$ surface for $0 \leqslant \xi \leqslant 1$ and $0 \leqslant \gamma \leqslant 3$.

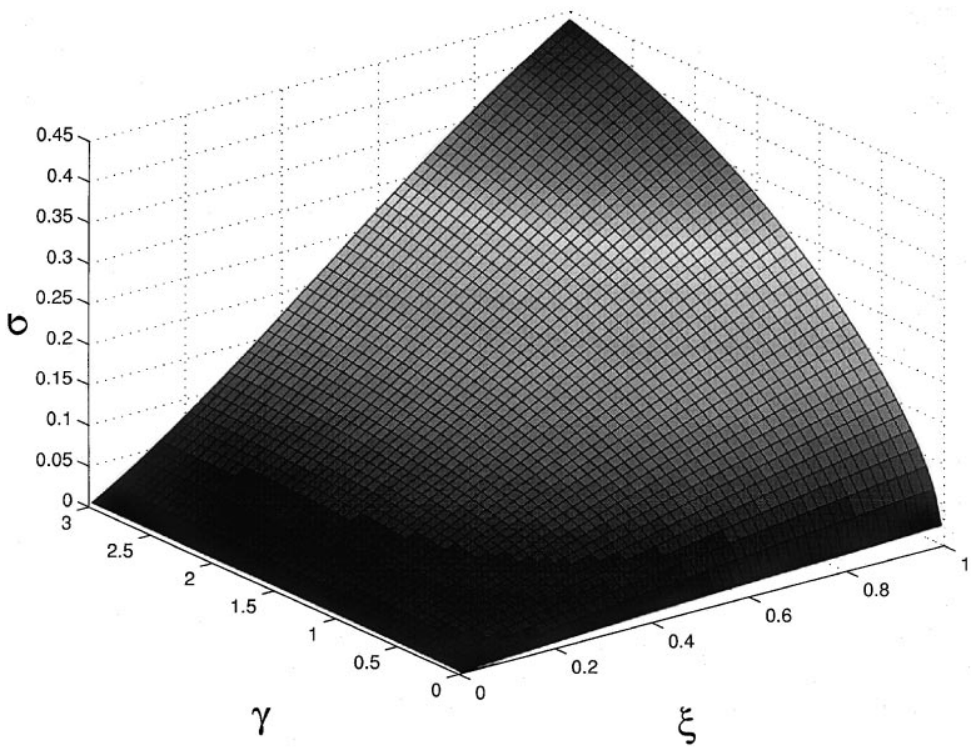

Fig. 2. $\sigma$ surface for $0 \leqslant \xi \leqslant 1$ and $0 \leqslant \gamma \leqslant 3$.

been previously developed using the screened Rutherford cross-section [10]. However, we have decided to employ a differential cross-section (DCS) with more physical content, one based on a partial-wave analysis.

\subsection{Generation of multiple-scattering angles}

DCSs for elastic scattering of electrons by neutral atoms have been calculated using the PWADIR code of Salvat and Mayol [11]. This 


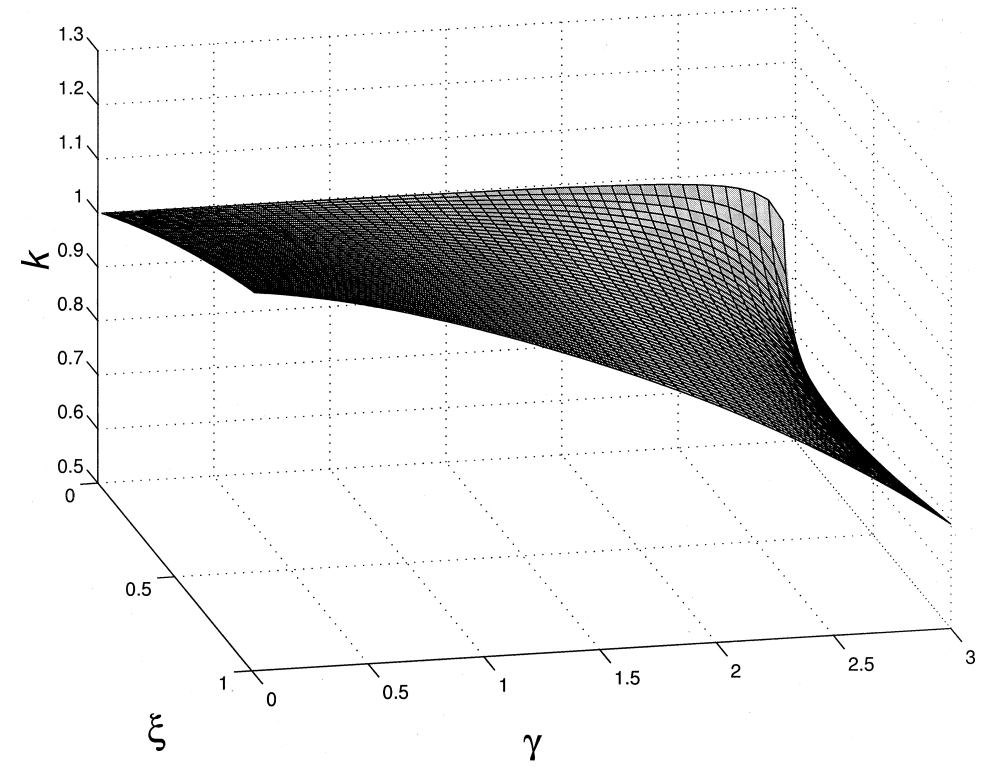

Fig. 3. $k$ surface for $0 \leqslant \xi \leqslant 1$ and $0 \leqslant \gamma \leqslant 3$.

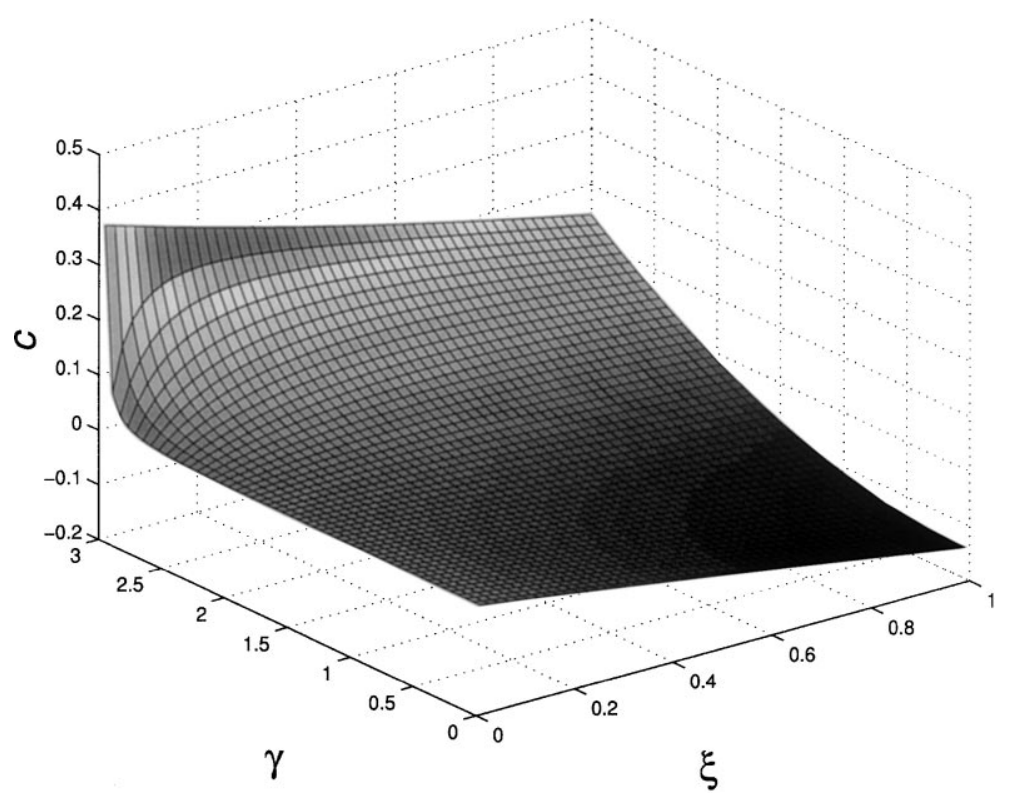

Fig. 4. $c$ surface for $0 \leqslant \xi \leqslant 1$ and $0 \leqslant \gamma \leqslant 3$.

code computes relativistic phase shifts from the numerical solution of the radial Dirac equation, for a given interaction field, and determines the corresponding DCS. We have adopted the parameterization of the Dirac-Hartree-Fock-Slater (DHFS) screened potential given by Salvat et al. 


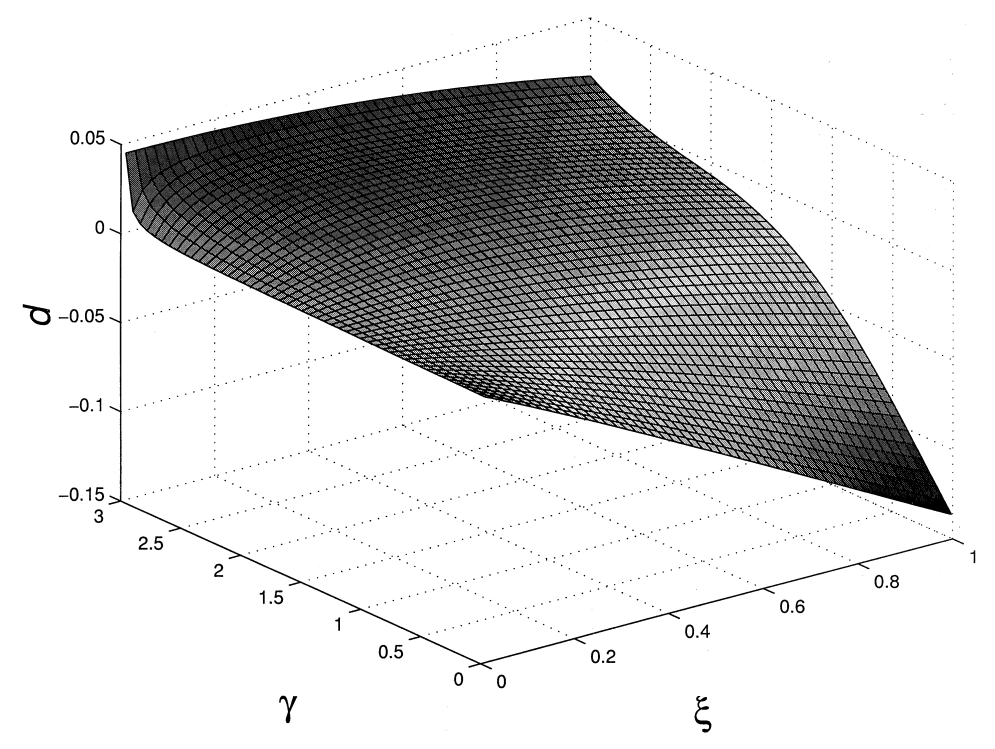

Fig. 5. $d$ surface for $0 \leqslant \xi \leqslant 1$ and $0 \leqslant \gamma \leqslant 3$.

[12], which leads to essentially the same DCSs as the numerical DHFS field. The effect of exchange between the projectile and the electrons in the target atoms has been accounted for by means of the approximate local field correction of Furness and McCarthy [13]. With this scheme, elastic DCSs can be calculated for incident electrons with energies up to a few $\mathrm{MeV}$. For higher energies, the numerical calculation becomes prohibitively lengthy and one must rely on approximate factorization methods. It should also be noted that the physical model (static field approximation) loses validity for projectiles with energies of the order of $1 \mathrm{keV}$ and less, since slow projectiles may cause appreciable polarization of the target atom. Fortunately, these energies are below the range of interest of most transport calculations. Our computer program generates a table of DCS values for a grid of about 600 scattering angles suitably distributed (logarithmically for small deflections and uniformly for large deflections). The DCS at other angles is obtained by linear interpolation. Computed DCSs for the elements $C(Z=6)$ and PB $(Z=82)$ and electrons and positrons with different energies are displayed in Figs. 6 and 7, respectively.

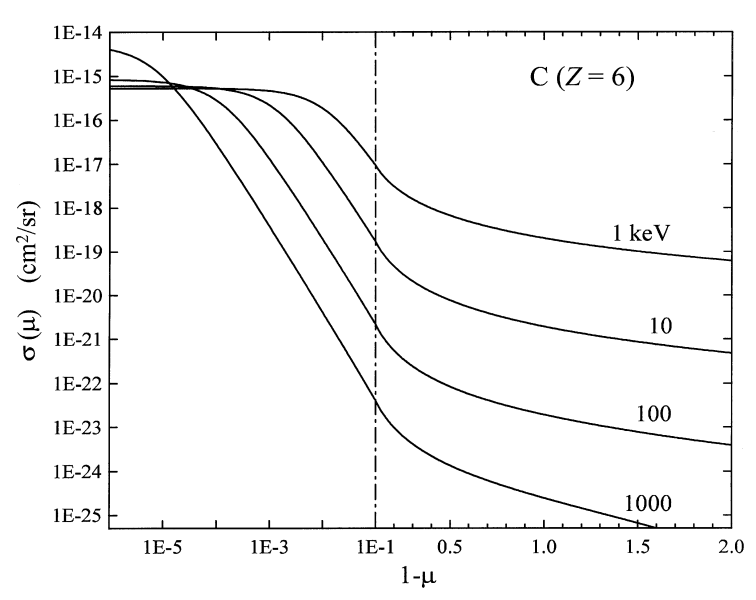

Fig. 6. Partial wave DCS for elastic scattering of electrons with the indicated kinetic energies by carbon atoms.

The moments $g_{\ell}$, Eq. (3), of the single-scattering distribution, disregarding energy loss, can be expressed as

$g_{\ell}=\frac{N_{\mathrm{A}} \rho}{A}\left(\sigma_{0}+f_{\ell}\right)$,

where

$\sigma_{0}=2 \pi \int_{-1}^{1} \mathrm{~d} \mu \sigma(\mu)$ 


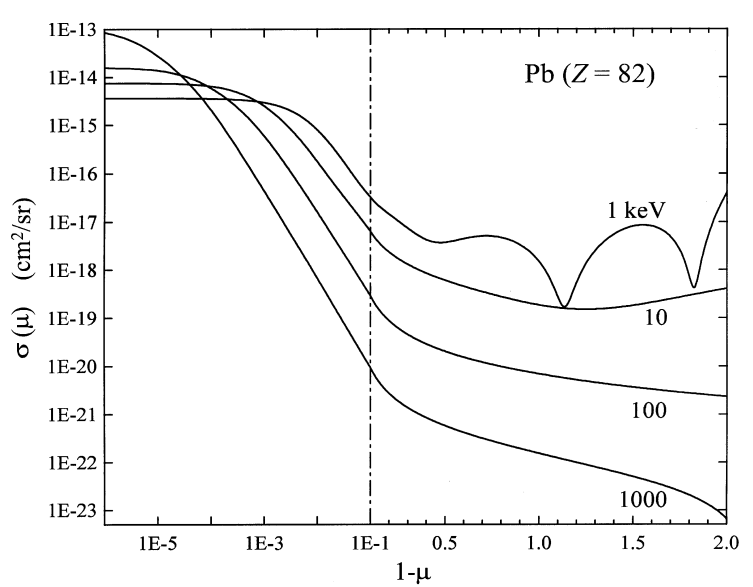

Fig. 7. Partial wave DCS for elastic scattering of electrons with the indicated kinetic energies by lead atoms.

is the total atomic cross-section and

$f_{\ell}=2 \pi \int_{-1}^{1} \mathrm{~d} \mu \sigma(\mu) P_{\ell}(\mu)$

The quantities $f_{\ell}$ have been calculated numerically from the partial-wave DCS by using the following algorithm. First, the integration interval $(-1,1)$ is split into a number of subintervals in such a way that the DCS varies by less than a factor of 10 within each subinterval. Then, a 500-point Gauss quadrature formula is used to evaluate the integral within each subinterval. Since the Legendre polynomials are generated by using the upward recursion relation, the algorithm can be coded to compute simultaneously all moments up to a given order, 600 in the present work. The calculation of these moments is very fast, a few seconds on a 366 $\mathrm{MHz}$ IBM compatible PC.

To check the accuracy of the calculated moments, we compared the original DCS with the result of adding up its Legendre expansion,

$\sigma(\mu)=\sum_{\ell=1}^{\infty} \frac{2 \ell+1}{4 \pi} f_{\ell} P_{\ell}(\mu)-\ell(\mu)$

For low-energy electrons, when the DCS is relatively wide and its Legendre series converges rapidly, this comparison is satisfactory. Differences between the original DCS and the "reconstructed" one are less than $0.001 \%$. In principle, the accuracy of the calculated moments should be independent of the energy, since the DCS varies slowly in each subinterval. The Goudsmit-Saunderson distribution is obtained by summing up its Legendre expansion (after removing the no-scattering part) and is expected to be as accurate as the reconstruction of the DCS, provided that the Legendre series actually does converge. At high energies, convergence with the 600 calculated moments is obtained only if the pathlength is large enough.

The random sampling of the scattering angle, from both the single-scattering DCS (analog simulation) and from the GS distribution (class I condensed simulation), is performed as follows. We start from a table of values of the corresponding probability distribution (not necessarily normalized) at the points of the angular grid mentioned above. To generate random deflection angles, we apply the inverse transform method to the (continuous) distribution obtained by linear interpolation within this table (i.e., the distribution of sampled values is done exactly from a piecewise linear distribution). With the aid of a binarysearch method, this sampling algorithm is very fast (about 300,000 random values generated per second on a $366 \mathrm{MHz}$ personal computer).

\subsection{Implementation of the transport mechanics}

In the numerical implementation of the algorithm, use is made of the fact that the azimuthal direction of either the scattering angle or the spatial displacement is arbitrary for unpolarized scattering. We adopted the following approach:

1. Start with an electron with initial position $\vec{x}_{0}$ and direction $\vec{\Omega}_{0}$ and sample the multiple-scattering angles $\Theta(s)$ (from the Goudsmit-Saunderson distribution) and $\Phi_{1}$ (uniformly in $(0,2 \pi)$ ).

2. Do a partial transport of the particle to the point at which the additional lateral straggling takes place. i.e.,

$$
\begin{aligned}
& x / s=f r \sin \Theta(s) \cos \Phi_{1}, \\
& y / s=f r \sin \Theta(s) \sin \Phi_{1}, \\
& z / s=k(1-r)+c+(k r+d) \cos \Theta(s),
\end{aligned}
$$


relative to an initial direction along the $\hat{z}$-axis but account, via rotation and translation, for the particle's actual direction and position.

3. Apply the additional lateral straggling

$$
\begin{aligned}
& \Delta x / s=\sigma \cos \Theta(s) \cos \Phi_{2}, \\
& \Delta y / s=\sigma \cos \Theta(s) \sin \Phi_{2}
\end{aligned}
$$

in a plane perpendicular to the particle's initial direction.

4. Rotate the particle's direction accounting for the scattering angles, $\Theta(s)$ and $\Phi_{1}$.

\section{Simulation results}

To demonstrate the quality of the new transport mechanics algorithm, we performed simulations of spatial and angular distributions of electrons with various energies after traveling different pathlengths in a number of elements. The simulations probed the practical limits of the $\gamma$ parameter and cross-section shape. However, here we will only present the graphical results for 100 $\mathrm{keV}$ electrons in $\mathrm{Pb}$ with pathlengths of 75 mean free paths (MFPs). This corresponds to $\gamma=2.185$ and $\xi=0.507$. Results from the new algorithm are compared here with those from equivalent analog (collision by collision) simulations using the same single-scattering DCS, which provide essentially exact results. The comparison also includes results from PENELOPEs transport mechanics, which were obtained for the same cases as the new mechanics.

In Figs. 8-10, we show the distributions for $z$, $r=\sqrt{x^{2}+y^{2}}$ and $x$. The results were obtained using $6 \times 10^{7}$ histories. The one-step distributions differ manifestly from the analog distributions, reflecting the somewhat artificial nature of the transport mechanics. The shapes of the distributions obtained from the new mechanics are generally closer to the analog distributions than the results from PENELOPEs mechanics; in particular the $p(r)$ distributions are peaked at finite values of $r$, in accordance with the analog results, whereas PENELOPE sets the most probable lateral displacement at $r=0$.
$100 \mathrm{keV}$ electrons in lead 75 mfp's, 1 step

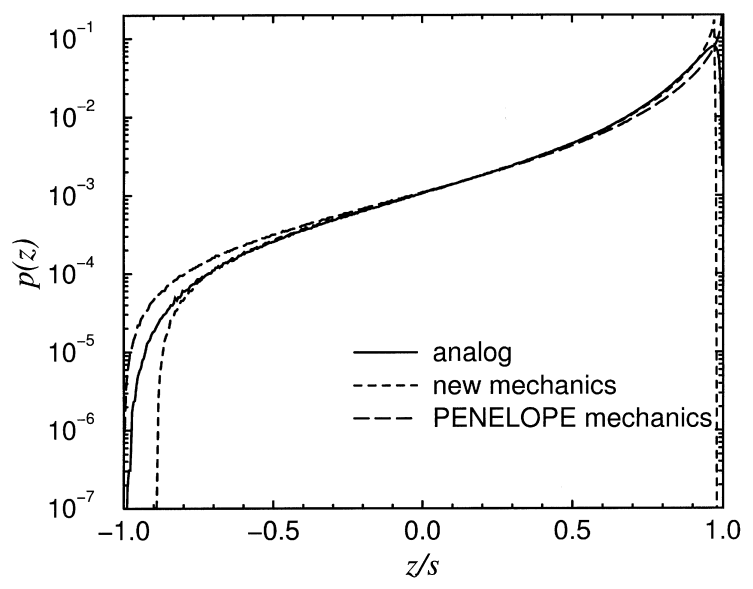

Fig. 8. The distribution of $z$ for $100 \mathrm{keV}$ electrons in lead taking a single-step of pathlength 75 elastic scattering MFPs.

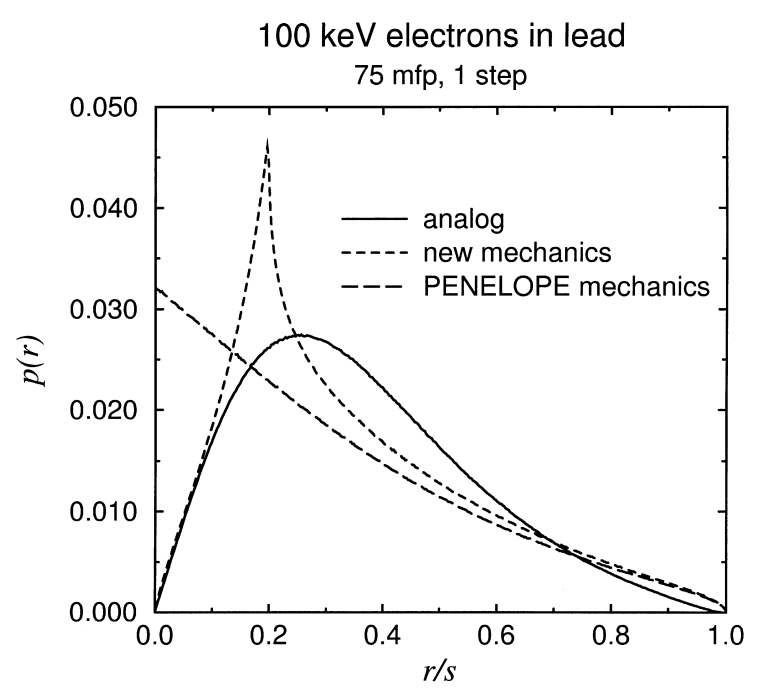

Fig. 9. The distribution of $r=\sqrt{x^{2}+y^{2}}$ for the simulation described in the caption of Fig. 8.

When the electron pathlength is split into two equal steps, the shapes of the simulated distributions improve substantially for both schemes. Figs. 11-13 display results of the two-step simulations for the same case studied above. Two-step distributions are twice as expensive to simulate than those with one step, but the extra cost is largely 


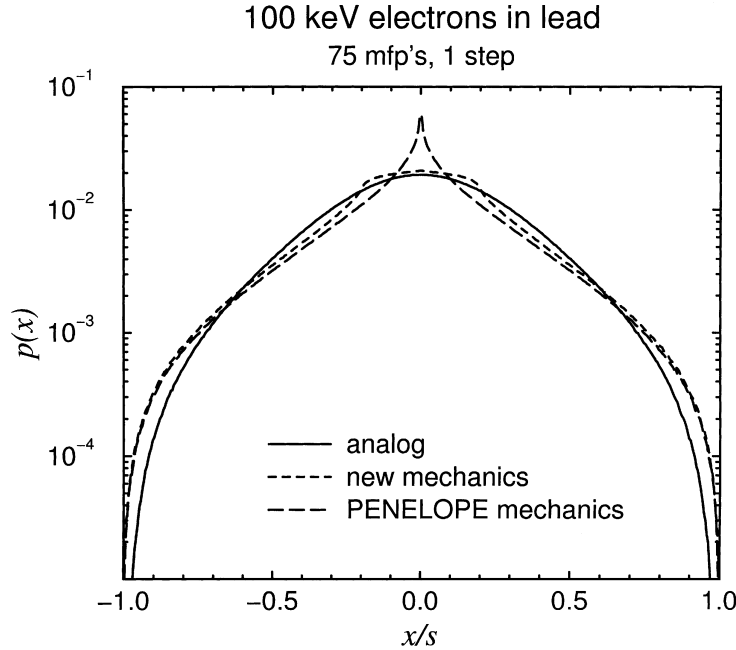

Fig. 10. The distribution of $x$ for the simulation described in the caption of Fig. 8.

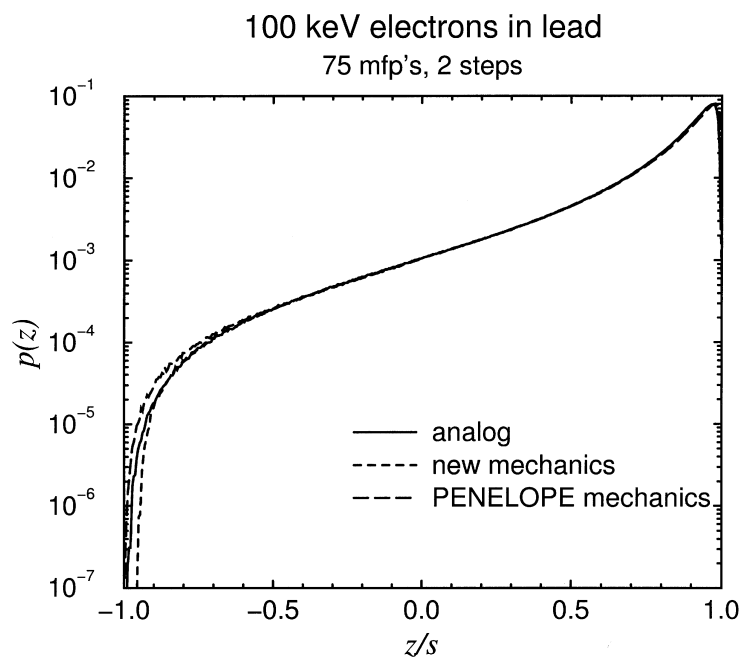

Fig. 11. The distribution of $z$ for $100 \mathrm{keV}$ electrons in lead taking two equal steps comprising a total pathlength of 75 elastic scattering MFPs. $6 \times 10^{7}$ histories were employed in this simulation. This case corresponds to $\gamma=2.185$ and $\xi=0.507$.

compensated by the gain in accuracy. It is satisfying that the crude details of the one-step distributions are almost completely washed out, for both the new transport mechanics and PENELOPE (which both use the the same physical information). The only visible artifact is the little bump

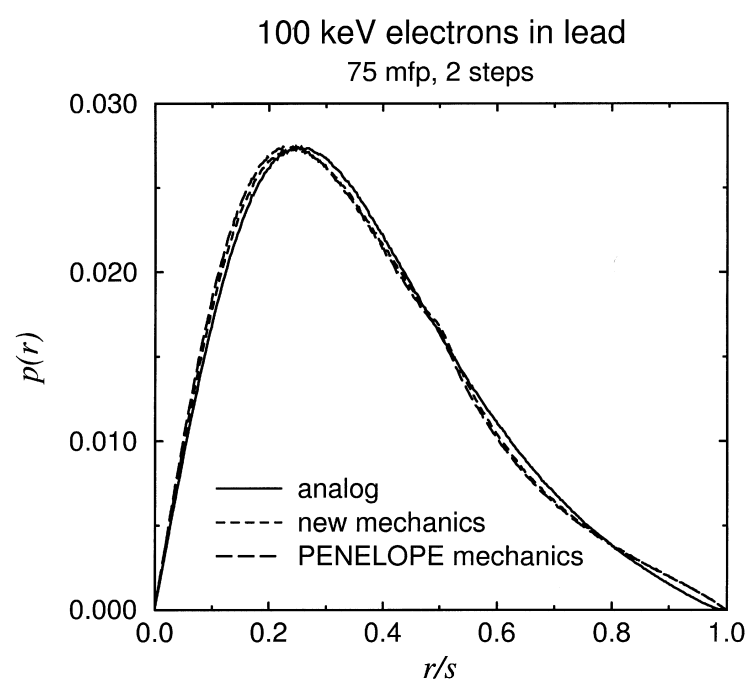

Fig. 12. The distribution of $r=\sqrt{x^{2}+y^{2}}$ for the simulation described in the caption of Fig. 11.

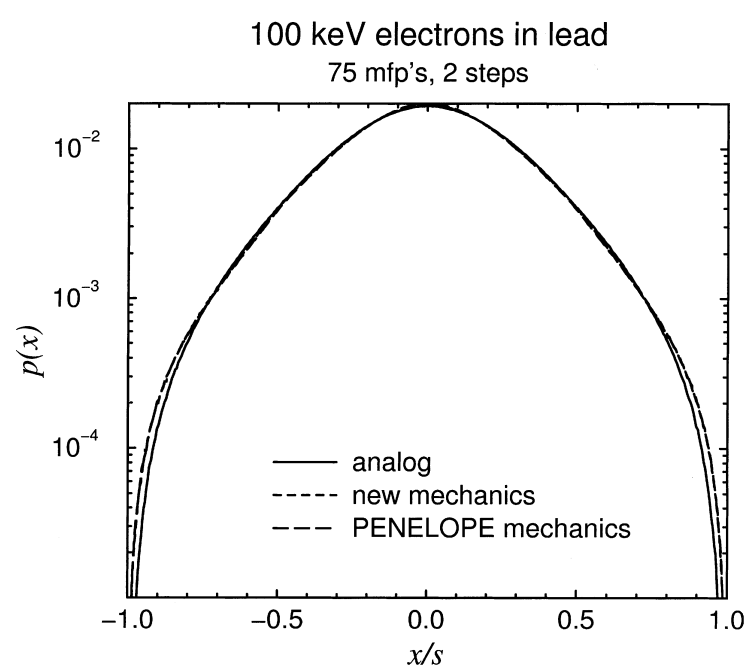

Fig. 13. The distribution of $x$ for the simulation described in the caption of Fig. 11.

in the lateral distributions, which disappears when the pathlength is split in four or more steps. These results do not imply that calculations with PENELOPEs and the new mechanics are equally accurate. Inspection of the spatial moments and space-angular correlations obtained by the two methods shows that the new mechanics is more 
Table 1

Spatial moments and space-angular correlations of the multiple-scattering distributions of $100 \mathrm{keV}$ electrons after traveling $75 \mathrm{MFPs}$ in lead $(\xi=0.507) . A \equiv\langle x \sin \Theta \cos \Phi+y \sin \Theta \sin \Phi\rangle^{\mathrm{a}}$

\begin{tabular}{|c|c|c|c|c|c|c|}
\hline & \multirow[b]{2}{*}{ Exact } & \multicolumn{4}{|c|}{ PENELOPE } & \multirow{2}{*}{$\frac{\text { New }}{1 \text { step }}$} \\
\hline & & 1 step & 2 steps & 4 steps & 6 steps & \\
\hline$\langle z\rangle$ & 58.83 & $60.08(2)$ & $59.14(2)$ & $58.91(2)$ & $58.87(2)$ & $58.82(2)$ \\
\hline$\left\langle z^{2}\right\rangle$ & 3840.0 & $4042.0(2)$ & $3888.0(2)$ & $3852.0(2)$ & $3846.0(2)$ & $3840.0(2)$ \\
\hline$\langle z \mu\rangle$ & 42.25 & $43.33(3)$ & $42.52(3)$ & $42.33(3)$ & $42.27(3)$ & $42.25(3)$ \\
\hline$A$ & 16.58 & $16.74(2)$ & $16.62(2)$ & $16.59(2)$ & $16.58(2)$ & $16.59(2)$ \\
\hline$\left\langle x^{2}+y^{2}\right\rangle$ & 943.4 & $837.0(10)$ & $932.2(10)$ & $942.4(10)$ & $943.1(10)$ & $943.9(10)$ \\
\hline Time (s) & & 3.8 & 7.8 & 15.6 & 23.4 & 10.6 \\
\hline
\end{tabular}

${ }^{a}$ Numbers in parentheses are statistical uncertainties $(3 \sigma)$ in units of the last significant figure of each value.

accurate and, therefore, it allows simulating a given pathlength in fewer steps.

This is illustrated in Table 1, where we give spatial moments and space-angular correlations for $100 \mathrm{keV}$ electrons in lead; the pathlength is half a transport mean free path (i.e., $\xi=0.507$ ); each simulation involved the generation of $10^{7}$ histories. We see that PENELOPEs mechanics gives results that deviate in the expected way, according to Eq. (8). In particular, it gives values of the longitudinal moments, $\langle z\rangle$ and $\left\langle z^{2}\right\rangle$, that are systematically too large, even when the pathlength is divided into six steps. On the other hand, the new mechanics gives the correct moments in a single step. The cost of implementing the new algorithm is indicated in the last line of the table, where we give the number of seconds to simulate $10^{6}$ histories on a $366 \mathrm{MHz}$ PII. We see that the computation cost of the new algorithm is about three times that of the random hinge in this particular example.

\section{Concluding remarks}

The new electron transport mechanics algorithm provides a more accurate description of spatial displacements than previous approaches. Although its accuracy can be matched by PENELOPEs mechanics, the latter will usually require splitting the pathlength into a larger number of steps. For pure elastic scattering, it has been shown that two-step simulations already yield fairly accurate space-angular distributions, which have their first moments correct (apart from statistical fluctuations).

In principle, the new algorithm could be improved, e.g. by straggling some of the parameters in Eq. (9), to yield qualitatively improved one-step distributions. However, this is far from trivial. In practice, it may be equally expedient (and probably faster) to appropriately increase the number of steps. It should be noted that in real simulations the number of steps per electron trajectory will be of the order of 10 or larger. Under these circumstances, the effect of any possible improvement of the transport mechanics on the final results will be hardly seen.

Although the present paper has been limited to pure elastic scattering for simplicity, the new transport algorithm can be readily combined with the continuous-slowing-down approximation to include energy losses. It is also particularly amenable for use in mixed, class II simulations, where it can allow increasing the cutoff angle (i.e. reducing the number of elastic hard events to be simulated) considerably. Work along these lines is in progress.

\section{Acknowledgements}

We gratefully acknowledge the numerous discussions with Prof. Ed Larsen (Department of Nuclear Engineering and Radiological Sciences, University of Michigan), Dr. José María Fernández-Varea (Universitat de Barcelona), 
Dr. Josep Sempau (Universitat Politècnica de Catalunya, Barcelona) and Mr. Ernest Benedito (Universitat de Barcelona) who kindly provided a fast calculational method for computing Legendre moments. Additionally, Mr. David Lorch (University of Michigan) is thanked for assistance with the figures. One of us (AFB) gratefully acknowledges partial financial support from LLNL and ADAC Laboratories (Milpitas, California) as well as a PVI grant from the University of Barcelona.

\section{References}

[1] S.A. Goudsmit, J.L. Saunderson, Phys. Rev. 57 (1940) 24. [2] S.A. Goudsmit, J.L. Saunderson, Phys. Rev. 58 (1940) 36. [3] H.W. Lewis, Phys. Rev. 78 (1950) 526.
[4] I. Kawrakow, A.F. Bielajew, Nucl. Instr. and Meth. B 142 (1998) 253.

[5] J. Baró, J. Sempau, J.M. Fernández-Varea, F. Salvat, Nucl. Instr. and Meth. B 100 (1995) 31.

[6] F. Salvat, J.M. Fernández-Varea, J. Baró, J. Sempau, PENELOPE, an algorithm and computer code for the Monte Carlo simulation of electron-photon showers, Informes Tecnicos CIEMAT n. 799, Centro de Investigaciones Energéticas, Medioambientales y Tecnológicas, Madrid, 1996.

[7] J. Sempau, E. Acosta, J. Baró, J.M. Fernández-Varea, F. Salvat, Nucl. Instr. and Meth. B 132 (1997) 377.

[8] I. Kawrakow, Nucl. Instr. and Meth. B 114 (1996) 307.

[9] E.W. Larsen, Ann. Nucl. Energy 19 (1992) 701.

[10] I. Kawrakow, A.F. Bielajew, Nucl. Instr. and Meth. B 134 (1998) 325.

[11] F. Salvat, R. Mayol, Comput. Phys. Commun. 74 (1993) 358.

[12] F. Salvat, J.D. Martínez, R. Mayol, J. Parellada, Phys. Rev. A 36 (1987) 467.

[13] J.B. Furness, I.E. McCarthy, J. Phys. B 6 (1973) 2280. 